\title{
Anti-Platelet Factor 4/Heparin Antibody Formation Occurs Endogenously and at Unexpected High Frequency in Polycythemia Vera
}

\author{
Sara C. Meyer, ${ }^{1,2,3}$ Eva Steinmann, ${ }^{2}$ Thomas Lehmann, ${ }^{1,2}$ Patricia Muesser, \\ Jakob R. Passweg, ${ }^{2}$ Radek C. Skoda, ${ }^{2,3}$ and Dimitrios A. Tsakiris ${ }^{1,2}$ \\ ${ }^{1}$ Diagnostic Hematology, Department of Laboratory Medicine, University Hospital Basel, Basel, Switzerland \\ ${ }^{2}$ Division of Hematology, Department of Medicine, University Hospital Basel, Basel, Switzerland \\ ${ }^{3}$ Experimental Hematology, Department of Biomedicine, University Hospital Basel, Basel, Switzerland \\ Correspondence should be addressed to Dimitrios A. Tsakiris; dimitrios.tsakiris@usb.ch
}

Received 3 February 2017; Revised 5 April 2017; Accepted 24 April 2017; Published 18 June 2017

Academic Editor: Zsuzsa Bagoly

Copyright (C) 2017 Sara C. Meyer et al. This is an open access article distributed under the Creative Commons Attribution License, which permits unrestricted use, distribution, and reproduction in any medium, provided the original work is properly cited.

\begin{abstract}
Background. Myeloproliferative neoplasms (MPN) encounter thromboses due to multiple known risk factors. Heparin-induced thrombocytopenia (HIT) is a thrombotic syndrome mediated by anti-platelet factor 4 (PF4)/heparin antibodies with undetermined significance for thrombosis in MPN. We hypothesized that anti-PF4/heparin Ab might occur in MPN and promote thrombosis. Methods. Anti-PF4/heparin antibodies were analyzed in 127 MPN patients including 76 PV and 51 ET. Screening, validation testing, and isotype testing of anti-PF4/heparin Ab were correlated with disease characteristics. Results. Anti-PF4/heparin antibodies were detected in $21 \%$ of PV and $12 \%$ of ET versus $0.3-3 \%$ in heparin-exposed patients. Validation testing confirmed anti-PF4/heparin immunoglobulins in $15 \%$ of PV and 10\% of ET. Isotype testing detected $9.2 \%$ IgG and 5.3\% IgM in PV and exclusively IgM in ET. IgG-positive PV patients encountered thromboses in 57.1\% suggesting anti-PF4/heparin IgG may contribute to higher risk for thrombosis in MPN. Overall, 45\% of PV patients experienced thromboses with 11.8\% positive for anti-PF4/heparin IgG versus 7.1\% in PV without thrombosis. Conclusion. Anti-PF4/heparin antibodies occur endogenously and more frequently in MPN than upon heparin exposure. Thrombotic risk increases in anti-PF4/heparin IgG-positive PV reflecting potential implications and calling for larger, confirmatory cohorts. Anti-PF4/heparin IgG should be assessed upon thrombosis in PV to facilitate avoidance of heparin in anti-PF4/heparin IgG-positive PV.
\end{abstract}

\section{Introduction}

Myeloproliferative neoplasms (MPN) are clonal disorders of hematopoiesis with excessive proliferation of mature myeloid cells. They comprise several related entities [1] including chronic myeloid leukemia (CML) characterized by translocation $t(9 ; 22)$ known as the Philadelphia chromosome and the classical, Philadelphia-negative MPN. These present as essential thrombocythemia (ET) characterized by thrombocytosis, polycythemia vera (PV) with predominant erythrocytosis and concomitant leuko- and thrombocytosis, or myelofibrosis (MF) with expansion of megakaryocytes and reactive bone marrow fibrosis [2]. More rarely, chronic neutrophilic leukemia (CNL) or chronic eosinophilic leukemia (CEL) are also seen [1]. PV, ET, and MF represent the focus of this study and are subsequently referred to as MPN. They are characterized by hyperactive signaling of the JAK2 kinase $[3,4]$ essentially involved in hematopoiesis [5] which is constitutively activated by acquired gain of function mutations. These driver mutations affect JAK2 itself, such as JAK2V617F in $95 \%$ of PV and $50 \%$ of ET and MF [6-9] and JAK2 exon 12 mutations in PV [10]. In addition, mutations in the thrombopoietin receptor MPL [11], such as MPLW515L, or in the chaperone protein calreticulin $(C A L R)$ were identified in ET and MF, which converge on activation of JAK2 signaling [12-15]. 
While MPN potentially transform to acute myeloid leukemia with dismal prognosis [16], the most frequent complications in MPN are thrombohemorrhagic events [17, 18]. They relevantly contribute to the substantial disease burden and their avoidance is a major goal of current therapies. Thrombotic events exceed the hemorrhagic complications in MPN which occur mainly due to depletion of ultralarge von Willebrand factor (VWF) multimers by thrombocytosis leading to acquired von Willebrand syndrome (VWS) and altered platelet function $[17,19]$. Thromboses in MPN affect both the arterial and the venous vascular beds comprising stroke or transient ischemic attacks, cardiac events, deep vein thrombosis, and pulmonary embolism as well as peripheral arterial or venous thrombosis [20]. Thromboses in MPN frequently present in atypical locations such as the splanchnic veins including mesenteric, splenic, portal, and hepatic vein thrombosis (Budd-Chiari syndrome) or cerebral venous sinuses. Thrombotic risk is highest in PV with $16-27 \%$ of patients affected by arterial and $7.4-11 \%$ by venous events $[21,22]$ and in ET with $10-50 \%$ of patients affected by a thrombotic complication within a decade from diagnosis [23]. Several risk factors have been identified and have been implemented in prognostic scores estimating thrombotic risk. While age over 60 years and history of thromboses represent the strongest predictors for thrombotic events [22, 24$30]$, the implication of leukocytosis, elevated hematocrit, and the JAK2V617F driver mutation have also been established. In addition, common cardiovascular risk factors as well as hereditary thrombophilia are considered relevant for overall risk of thromboses in MPN [17]. The pathogenesis of thromboses in MPN is currently perceived as multifactorial since general and disease-specific prothrombotic factors coincide. Thrombocytosis which is a characteristic presenting feature in ET, PV, and prefibrotic forms of MF has not been validated as a thrombotic risk factor in MPN.

The panoply of factors currently known to contribute to thrombotic risk in MPN might not be exhaustive and additional effects should be considered. Heparin-induced thrombocytopenia (HIT) represents a rare thrombotic syndrome mediated by an immune response to platelet factor 4 (PF4) secreted from platelet alpha granules [31]. PF4 complexes with heparin when the latter is administered for treatment or prophylaxis of thrombosis, giving rise to an immunogenic neoantigen. Consecutive antibody formation against PF4/heparin complexes is seen in $0.3-3 \%$ of patients on heparin treatment [32] and mediates activation and clearance of platelets leading to thrombocytopenia and potentially thrombosis, the full manifestation of HIT. Anti-PF4/heparin immunoglobulin production ceases upon withdrawal of heparin and anti-PF4/heparin antibodies subsequently become undetectable after heparin treatment is stopped. Even after overt HIT, anti-PF4/heparin antibodies are undetectable by 50-80 days [33]. Differential effects of several types of heparin are known such as increased risk of anti-PF4/heparin antibody formation by unfractionated heparin (UFH) as compared to low molecular weight heparin (LMWH) [34]. In addition, increased levels of PF4 in settings of platelet activation or high turnover have been shown to promote anti-PF4/heparin antibody formation probably via increased abundance of antigen [31]. Inflammatory stimuli by bacterial infection $[35,36]$ or by tissue damage upon surgery or major trauma are also considered promoting development of HIT $[37,38]$. Interestingly, a recent study in orthopedic patients after knee or hip arthroplasty reported anti-PF4/heparin antibody formation in the absence of heparin treatment which was enhanced by dynamic versus static compression therapy for thromboprophylaxis $[39,40]$.

Insight into the significance of HIT for thrombosis in MPN is limited. The risk for thrombotic complications in MPN patients entails a high probability of heparin exposure during the course of the disease which could thereby put MPN patients at increased risk of developing HIT. However, diagnosis of HIT which includes substantial thrombocytopenia or a $50 \%$ fall in platelet counts is impeded by elevated baseline platelet levels in PV and ET. Thrombocytosis in MPN might mask occurrence of thrombocytopenia due to development of HIT, thereby leading to false negative assessments. Importantly, thrombocytosis in PV and ET is reflective of excessive platelet production and turnover, and platelets in MPN are known to circulate in an activated state, thus providing ample PF4 which could promote the formation of anti-PF4/heparin antibodies in PV and ET [17]. A limited number of PV and ET patients with occurrence of HIT have been reported (Table 1) [41-53]. In addition, analysis of a cohort of HIT patients found overrepresentation of ET and PV with 4.7\% (2/42) [54]. The true incidence of HIT in PV and ET has not been clearly assessed given the masking of thrombocytopenia by excessive platelet production and the scarcity of the literature on this topic limited to a handful of case reports and series. A comprehensive case series studying 29 MPN patients [41] observed strong clinical evidence for HIT in five patients, but only two were tested for anti-PF4/ heparin antibodies. Therefore, we employed a systematic approach to assess PF4/heparin antibody formation and its significance for thrombosis in a large cohort of PV and ET patients given their high thrombotic risk. We hypothesized that anti-PF4/heparin antibodies could be prevalent in these patients with excessive platelet production and turnover and might contribute to thrombotic risk in MPN.

\section{Materials and Methods}

2.1. Characterization of Patients. A cohort of 127 MPN patients including $76 \mathrm{PV}$ and $51 \mathrm{ET}$ patients from our tertiary care center diagnosed according to the WHO classification were retrospectively analyzed for MPN disease characteristics. Blood counts including hematocrit, hemoglobin concentration, platelet counts, white blood cell (WBC), and neutrophil counts were assessed on an ADVIA hemocytometer. The JAK2V617F mutation was assessed by allele-specific PCR in peripheral blood granulocyte DNA. Patient histories were assessed for occurrence, number, and localization of thrombotic events which were diagnosed by duplex sonography for deep vein thrombosis or CT angiography for pulmonary embolism and arterial events. Splenomegaly was assessed by clinical examination, sonography, or CT scan. Informed consent was available from all individuals and approval from the local ethics committee was obtained. 


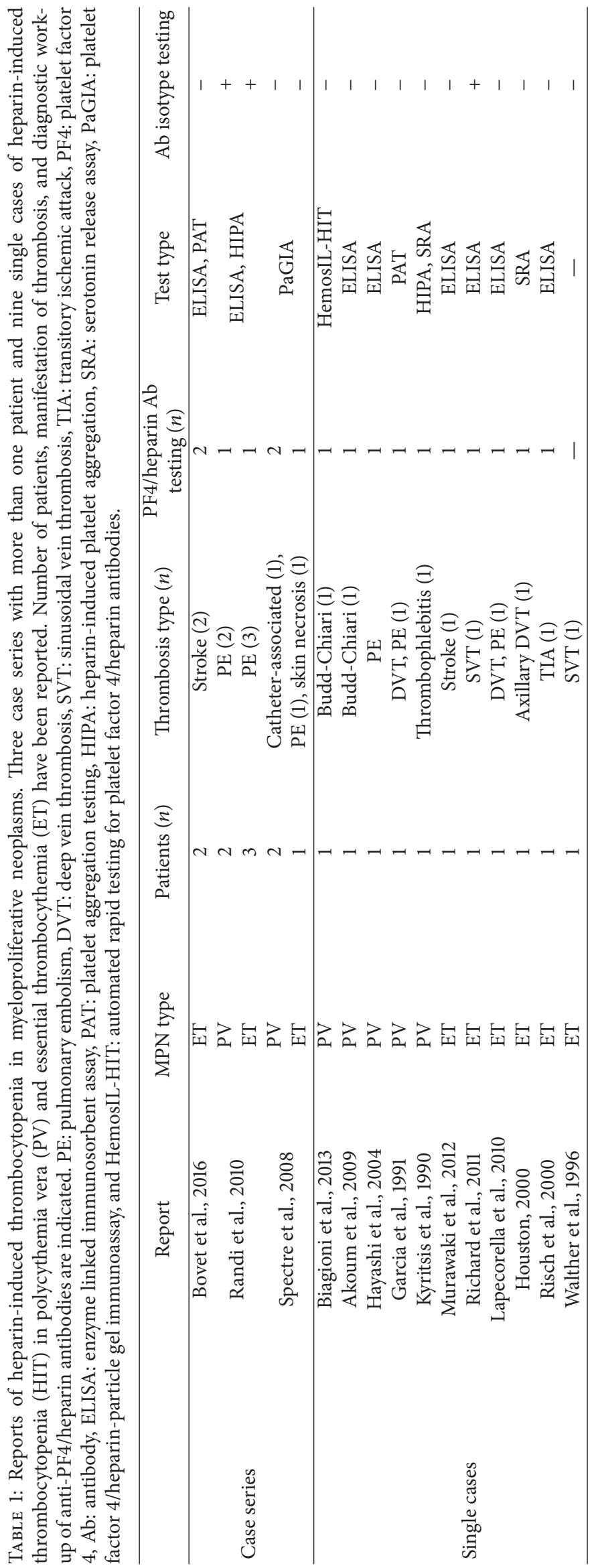


TABLE 2: Baseline characteristics of MPN patient cohort. Characteristic parameters of the study population of 127 MPN patients are displayed (mean for age and peripheral blood counts, frequency for all other parameters). Frequencies are indicated in percent of total patients: absolute numbers are given in parentheses.

\begin{tabular}{|c|c|c|}
\hline MPN type & Polycythemia vera & Essential thrombocythemia \\
\hline Patient cohort & \multicolumn{2}{|c|}{ total $n=127$} \\
\hline Patients $(n)$ & 76 & 51 \\
\hline Male $(n)$ & 42 & 18 \\
\hline Female $(n)$ & 34 & 33 \\
\hline \multicolumn{3}{|l|}{ Parameters at diagnosis } \\
\hline Age $(y)$ & 55.8 & 53.2 \\
\hline Hematocrit (\%) & 53.2 & 41.6 \\
\hline Hemoglobin (g/l) & 176.2 & 139.2 \\
\hline Platelets (G/l) & 602.9 & 984.3 \\
\hline Leukocytes (G/l) & 12.4 & 9.0 \\
\hline Splenomegaly (\%) & $55.3(42 / 76)$ & $41.2(21 / 51)$ \\
\hline$J A K 2 \mathrm{~V} 617 \mathrm{~F}(\%)$ & $84.2(64 / 76)$ & $52.9(27 / 51)$ \\
\hline \multicolumn{3}{|c|}{ Thrombohemorrhagic events } \\
\hline Thrombosis & $44.7(34 / 76)$ & $39.2(20 / 51)$ \\
\hline Hemorrhage & $18.4(14 / 76)$ & $3.9(2 / 51)$ \\
\hline \multicolumn{3}{|l|}{ Medications } \\
\hline Antiaggregation & $94.7(72 / 76)$ & $88.2(45 / 51)$ \\
\hline Oral anticoagulation & $30.3(23 / 76)$ & $11.8(6 / 51)$ \\
\hline Cytoreduction & $75.0(57 / 76)$ & $68.6(35 / 51)$ \\
\hline
\end{tabular}

2.2. Anti-PF4 Antibody Testing. Testing for anti-PF4 immunoglobulins was performed in PV and ET patient serum samples stored at $-80^{\circ} \mathrm{C}$. We employed a sequential approach including a screening and a subsequent validation assay in positive samples for optimized specificity. Initial screening for anti-PF4 antibodies was performed by a commercial anti-PF4 ELISA globally detecting IgG, IgA, and IgM antibodies (ZYMUTEST HIA IgGAM ELISA, product number RK040D). Positive samples were subsequently subjected to specific isotype testing for validation and for determination of IgG, IgM, and IgA isotypes (ZYMUTEST HIA IgG, IgA, IgM, product number RK040E). IgG subclasses and FcgRIIa H131R polymorphism were not specifically assessed in the study.

2.3. Statistical Analysis. Statistical analysis was performed by SPSS software. Pearson's X2 test (two-by-two table) was used to compare categorical variables. Significance level was set at $p<0.05$ in two-sided tests.

\section{Results}

3.1. Baseline Characterization of MPN Patient Cohort. The study population of 127 individuals consisted of $76 \mathrm{PV}$ and 51 ET patients. Baseline characteristics are shown in Table 2. Among PV patients, there was a slight male predominance ( $n=42$ male, $n=34$ female), while ET affected more women ( $n=18$ male, $n=33$ female). Mean age at diagnosis was 55.8 years in PV and 53.2 years in ET. PV patients were characterized by erythrocytosis reflected by increased red cell parameters (mean hematocrit 53.2\%, mean hemoglobin $176.2 \mathrm{~g} / \mathrm{l}$ ) as well as mild leukocytosis with mean white blood cell count (WBC) of $12.4 \mathrm{G} / \mathrm{l}$. Red cell parameters and WBC were within normal range in ET patients, who displayed pronounced thrombocytosis with mean platelet count of 984.3 G/1. Thrombocytosis was also present in PV (mean platelet count $602.9 \mathrm{G} / \mathrm{l})$. JAK2V617F was detected in $84.2 \%$ of PV and $52.9 \%$ of ET patients concordant with previous studies [6-9]. Splenomegaly by clinical assessment or imaging was more prevalent in PV (55.3\%) than ET (41.2\%). Thrombohemorrhagic complications were frequent with $63.1 \%$ in PV and $43.1 \%$ in ET. Thereof, $18.4 \%$ and $3.9 \%$ of patients suffered from bleeding events, respectively, while thromboses clearly outweighed bleeding events both in PV and in ET. Nearly all patients were on antiaggregatory prophylaxis with low dose aspirin (94.7\% in PV, $88.2 \%$ in ET), while much less individuals had received anticoagulation with vitamin $\mathrm{K}$ antagonists during the course of their disease $(30.3 \%$ in PV, $11.8 \%$ in ET). Substantial proportions had received cytoreductive therapy with $75.0 \%$ in PV and $68.6 \%$ in ET patients (Table 2). These analyses match well with established disease characteristics in PV and ET [2] and demonstrate that this patient population is representative and well suitable for investigations into the role of anti-PF4 immune responses in MPN.

3.2. Thromboses Are Frequent in PV and ET and Relate to Multiple Risk Factors. Thromboses occurred in 34/76 PV patients $(44.7 \%)$ and $20 / 51 \mathrm{ET}$ patients $(39.2 \%)$ during the course of disease highlighting the very substantial contribution of thrombotic events to disease burden in MPN. 
TABLE 3: Site of thrombotic events in MPN. The site of thromboses in $127 \mathrm{MPN}$ patients is displayed highlighting the relevance of thrombotic complications for disease burden in MPN. CNS: central nervous system, VTE: venous thromboembolism, DVT: deep vein thrombosis, PE: pulmonary embolism, and PAD: peripheral arterial disease.

\begin{tabular}{lcc}
\hline Site of thrombosis & \multicolumn{3}{c}{ Frequency } \\
\hline Total & 100 & $n$ \\
CNS & 37.0 & 54 \\
VTE & & 20 \\
$\quad$ DVT & 20.4 & \\
PE & 11.1 & 11 \\
Cardiac & 14.8 & 6 \\
Splanchnic & 13.0 & 8 \\
PAD & 3.7 & 7 \\
\hline
\end{tabular}

The slightly higher incidence of thrombotic complications in $\mathrm{PV}$ as compared to ET patients is in accordance with previous reports $[20,21]$ (Table 2 ). In the presented patient population, cerebrovascular events were the most prevalent manifestation of thrombosis in $37.0 \%$ of patients, followed by venous thromboembolism in $31.5 \%$ including $20.4 \%$ of deep vein thromboses, $11.1 \%$ of pulmonary embolism, and $14.8 \%$ with cardial ischemic events (Table 3). Splanchnic thromboses which are characteristic for MPN were seen in $7 / 54(13.0 \%)$ patients which represents a high incidence given their overall rarity. Two patients (3.7\%) with peripheral arterial disease and additional vascular risk factors such as previous cigarette smoking, arterial hypertension, and dyslipidemia showed peripheral arterial occlusions. A substantial proportion (19/54 patients) suffered from repeated thrombotic events with 3 patients even showing multiple ( 3 and more) thromboses during the disease course. Platelet counts $>400 \mathrm{G} / 1$ did not significantly affect thrombotic risk $(p=0.106)$, whereas patients with hematocrit $>0.46$ showed a significantly increased incidence of thromboses $(p=$ $0.010)$, as did patients with leukocytosis $>10 \mathrm{G} / 1(p=0.033)$ reflecting established risk factors [17].

3.3. Anti-PF4/Heparin IgG Antibodies Occur at High Frequency in PV Patients. Qualitative screening for anti-PF4/ heparin antibodies was positive in 22 individuals (17.3\%) of the entire cohort of 127 patients. Incidence in PV patients was higher than in ET with $21.0 \%$ tested positive in PV and $11.8 \%$ among ET patients (Table 4). Positive patients underwent subsequent validation testing, which confirmed anti-PF4/heparin antibodies in $14.5 \%$ of PV and $9.8 \%$ of ET patients (Figure 1). Immunoglobulin isotype testing showed that $9.2 \%$ of PV patients had circulating IgG against PF4, for which a functional role as platelet activators in pathogenesis of HIT has been demonstrated [31]. The prevalence of anti-PF4/heparin IgG among PV patients in our cohort is clearly higher than in patients treated with unfractionated heparin, for whom anti-PF4/heparin IgG have been reported in $0.3-3 \%$ after a treatment duration of more than four days [32] or at lower frequencies when LMWH is used [34].
Sequential antibody testing and isotype testing in 3/7 IgGpositive PV patients revealed persistent positivity after 847 , 674 , and 182 days, respectively, which supports the notion that anti-PF4/heparin IgG immunoglobulins can occur endogenously in PV patients and that antibody formation can be maintained despite the absence of concurrent heparin exposure. Immunoglobulin isotype testing also identified anti-PF4/heparin IgM in 5.3\% of PV patients. A thrombogenic potential of anti-PF4/heparin IgM has not been clearly established and a causative implication for thrombosis in HIT remains on debate. All anti-PF4/heparin antibodies detected in ET patients were of IgM isotype at a prevalence of $9.8 \%$. No anti-PF4/heparin IgG were observed in ET patients in our cohort. As patients with ET showed more pronounced thrombocytosis than PV patients, the absence of IgG isotypes in ET suggests that excessive platelet production associated with increased circulating levels of PF4 may not be the sole factor facilitating anti-PF4 immune responses in MPN but that additional promoting factors are at play specifically in PV patients (Table 4). However, it is noteworthy that antiPF4/heparin IgM antibodies persisted in a majority (5/9) of positive patients with 3/4 PV and 2/5 ET patients showing detectable IgM at two sequential assessments with intervals of $1003,904,811,539$, and 42 days. As several cases of clinically manifest HIT in ET patients have been reported (Table 1) and exclusively anti-PF4/heparin IgM were detectable in ET patients of our cohort, a potential implication of antiPF4/heparin IgM for platelet activation in the setting of MPN with thrombocytosis may warrant further evaluation in functional studies.

3.4. Anti-PF4/Heparin IgG Antibodies Confer a Tendency for Increased Thrombotic Risk in PV. We next assessed to what extent anti-PF4/heparin IgG immunoglobulins with the known potential to activate platelets in the pathogenesis of clinical HIT would contribute to thrombotic complications in PV. We observed that $57.1 \%$ of PV patients with circulating anti-PF4 IgG suffered from a thrombotic event at least once during the course of disease (Table 5) including both arterial and venous events according to the known spectrum of thromboembolic complications in MPN (Table 6). AntiPF4/heparin IgG-negative PV patients encountered thromboses in $43.5 \%$, reflecting a $31 \%$ increase in relative thrombotic risk for PV patients with anti-PF4/heparin IgG as compared to IgG-negative PV in our cohort (Table 5, $p>0.05$ ). Predictors of whether IgG-positive PV patients would actually develop thrombosis were absent suggesting that thrombosis in $\mathrm{PV}$ is a strongly multifactorial process, while the Fc $\gamma$ RIIa H131R polymorphism was not assessed in this study. When PV patients with a positive history of thrombosis were assessed for anti-PF4 immune responses, we observed that $11.8 \%$ had anti-PF4/heparin IgG antibodies, as compared to $7.1 \%$ IgG positivity in PV patients who never had a thrombosis. Although the increased risk for thrombotic events in PV patients with anti-PF4/heparin IgG is statistically nonsignificant in our cohort, a subtle but relevant contribution of anti-PF4/heparin IgG to thrombosis in PV cannot be excluded at this point, particularly in view of the multifactorial nature of thrombosis in MPN with hematocrit 
TABLE 4: Characterization of anti-PF4/heparin antibody formation in MPN. A cohort of 127 patients with myeloproliferative neoplasms (MPN) including polycythemia vera (PV, $n=76)$ and essential thrombocythemia (ET, $n=51)$ were characterized for anti-PF4/heparin antibodies. Analysis by a screening ELISA was subsequently validated by follow-up testing and antibody isotype testing determining IgG, IgM, and IgA. PF4: platelet factor 4; Ig: immunoglobulin.

\begin{tabular}{|c|c|c|c|c|c|c|}
\hline \multicolumn{2}{|c|}{ MPN } & \multicolumn{5}{|c|}{ PF4/heparin antibody characterization } \\
\hline \multirow{2}{*}{ Subtype } & \multirow{2}{*}{ Patients $(n)$} & \multirow{2}{*}{ Screening (\% positive) } & \multirow{2}{*}{ Validation (\% positive) } & \multicolumn{3}{|c|}{ Isotype testing (\% positive) } \\
\hline & & & & $\operatorname{IgG}$ & $\operatorname{IgM}$ & $\operatorname{IgA}$ \\
\hline PV & 76 & $21.1(16 / 76)$ & $14.5(11 / 76)$ & $9.2(7 / 76)$ & $5.3(4 / 76)$ & $0.0(0 / 76)$ \\
\hline ET & 51 & $11.8(6 / 51)$ & $9.8(5 / 51)$ & $0.0(0 / 51)$ & $9.8(5 / 51)$ & $0.0(0 / 51)$ \\
\hline Total & 127 & & & & & \\
\hline
\end{tabular}

TABLE 5: Impact of anti-PF4/heparin antibodies on thrombotic risk in polycythemia vera. Anti-PF4/heparin antibodies of IgG isotypes, which have a known implication in pathogenesis of HIT, were assessed for a potential impact on thrombotic risk in polycythemia vera. IgG positivity conferred a $31 \%$ increase of relative risk for thrombosis as compared to IgG-negative PV. Statistical significance was not reached due to sample number. PF4: platelet factor 4; Ig: immunoglobulin.

\begin{tabular}{lccc}
\hline & \multicolumn{2}{c}{ Anti-PF4/heparin IgG isotype } & Relative thrombotic risk \\
& Positive & Negative & $p$ value \\
\hline Thrombotic complications (\%) & 57.1 & 43.5 & 1.31 \\
No thrombotic complications (\%) & 42.9 & 56.5 & $>0.05$ \\
\hline
\end{tabular}

TABLE 6: Thromboembolic complications in anti-PF4/heparin IgG-positive PV patients. Thromboembolic complications are indicated in 7 PV patients positive for anti-PF4/heparin antibodies of IgG isotype. Thromboembolic events occurred in patients 1-4 and not in patients 5-7. Both arterial and venous complications as well as repeated events were seen. Splenic infarcts as in patient 5 are not considered classic thromboembolic complications in MPN. Grade IV PAD induced critical ischemia in patient 6 without an acute occlusive event. Pat: patient, Dgn: diagnosis, DVT: deep vein thrombosis, TIA: transitory ischemic attack, CVI: cerebrovascular infarction, NIHSS: National Institute of Health Stroke Scale, PE: pulmonary embolism, CHD: coronary heart disease, and PAD: peripheral arterial disease.

\begin{tabular}{|c|c|c|c|c|c|c|c|}
\hline Pat & Age at Dgn & Sex & MPN type & $\begin{array}{c}\mathrm{PF} 4 / \text { heparin } \mathrm{Ab} \\
\text { isotype }\end{array}$ & $\begin{array}{l}\text { Thromboembolic } \\
\text { complications }(n)\end{array}$ & 1st event & 2nd event \\
\hline 1 & 71 & $\mathrm{~m}$ & PV & $\operatorname{IgG}$ & 2 & $\begin{array}{l}\text { 2-level DVT with } \\
\text { postthrombotic } \\
\text { syndrome }\end{array}$ & TIA \\
\hline 2 & 49 & $\mathrm{~m}$ & PV & IgG & 2 & $\begin{array}{c}\text { CVI } \\
\text { (NIHSS } 5 \text { pt) }\end{array}$ & $\begin{array}{c}\text { CVI } \\
\text { (NIHSS } 16 \mathrm{pt})\end{array}$ \\
\hline 3 & 57 & $\mathrm{~m}$ & PV & IgG & 1 & $\begin{array}{l}\text { Bilateral PE with } \\
\text { pulmonal-arterial } \\
\text { hypertension }\end{array}$ & - \\
\hline 4 & 33 & $\mathrm{~m}$ & PV & $\operatorname{IgG}$ & 1 & $\begin{array}{l}\text { In-stent thrombosis } \\
\text { in early onset CHD }\end{array}$ & - \\
\hline 5 & 41 & $\mathrm{~m}$ & PV & IgG & 0 & $\begin{array}{l}\text { Splenic infarcts, no } \\
\text { splenic vein } \\
\text { thrombosis }\end{array}$ & - \\
\hline 6 & 61 & $\mathrm{~m}$ & PV & $\operatorname{IgG}$ & 0 & $\begin{array}{l}\text { Critical ischemia of } \\
\text { lower extremity in } \\
\text { grade IV PAD }\end{array}$ & - \\
\hline 7 & 69 & $\mathrm{f}$ & $\mathrm{PV}$ & IgG & 0 & - & - \\
\hline
\end{tabular}

and leukocyte levels as major risk factors. Further studies of more extensive patient populations are warranted to confirm the tendency for increased thrombotic risk in PV patients with anti-PF4/heparin IgG antibodies which we observe in our study. However, as PV is a rare hematological disorder, this will eventually require multicenter collaborative efforts.

Overall, we find a high frequency of circulating antiPF4/heparin IgG antibodies in patients with PV which are detectable in the absence of concurrent exposure to heparin. There is a tendency for increased thrombotic complications in PV patients positive for anti-PF4/heparin IgG. Due to the retrospective nature of this study, data on antibody positivity at the time of thrombosis is not available for our cohort. However, our data suggest that PV patients should undergo anti-PF4/heparin IgG testing as soon as a thrombotic event occurs to prevent potential boosting of the anti-PF4/heparin 


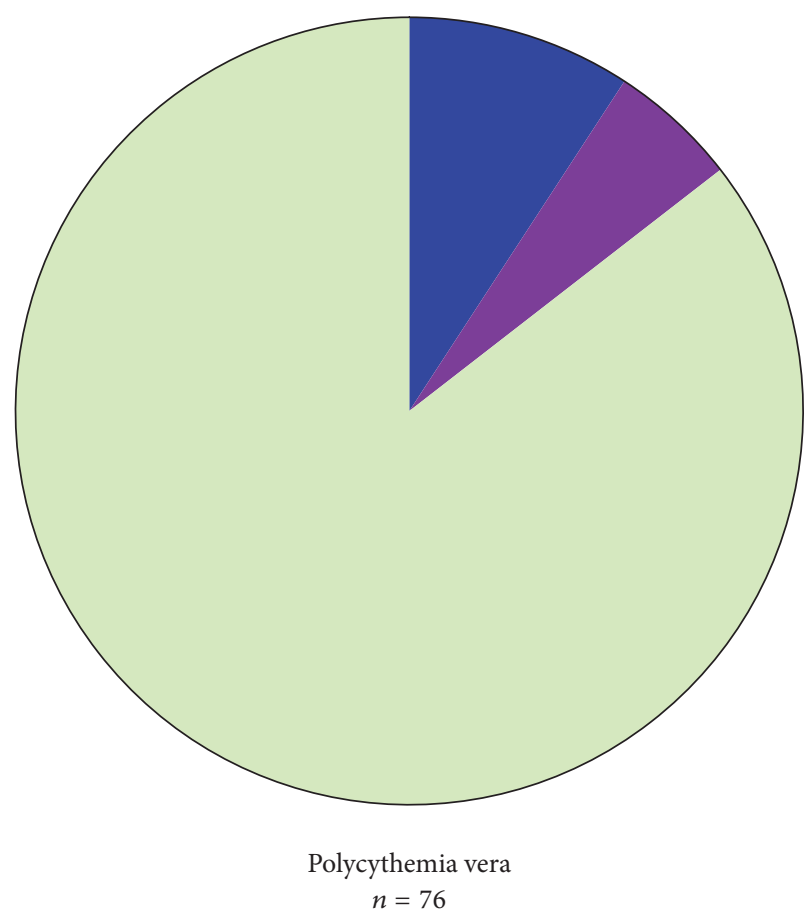

Anti-PF4/heparin IgG Anti-PF4/heparin IgM None

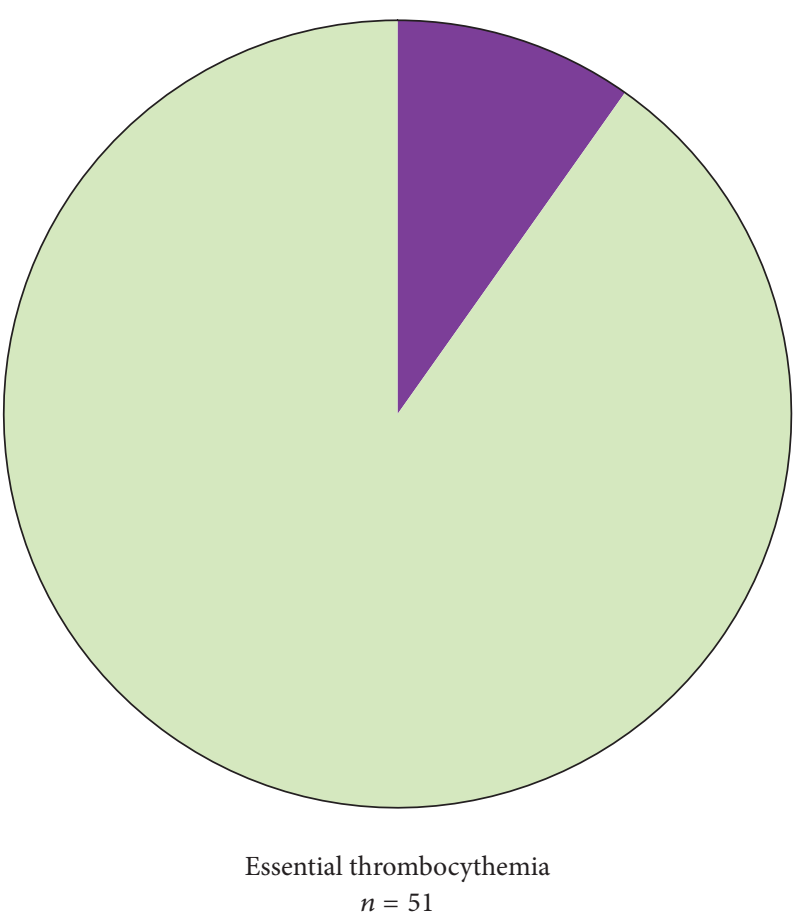

Anti-PF4/heparin IgM None

(a)

(b)

FIGURE 1: Endogenous anti-PF4/heparin IgG and IgM antibodies occur in polycythemia vera and essential thrombocythemia. (a) Anti$\mathrm{PF} 4 /$ heparin antibodies of IgG isotype are detected at considerable frequency in polycythemia vera (PV), while IgM isotype antibodies are also found. (b) Anti-PF4/heparin antibodies in essential thrombocythemia are of IgM isotype in our cohort. No IgA isotypes were detected. PF4: platelet factor 4; Ig: immunoglobulin.

immune response by initiation of heparin treatment as seen in rare reported cases [55] and to facilitate alternative anticoagulation in IgG-positive PV patients.

\section{Discussion}

We report on the prevalence of anti-PF4/heparin antibodies in a large cohort of PV and ET patients and evaluate to what extent anti-PF4/heparin IgG known to mediate platelet activation and consequent thrombosis in HIT would contribute to thrombosis in MPN. MPN are characterized by an acquired prothrombotic condition with multifaceted pathogenesis of interplaying proaggregatory and procoagulatory factors [17]. As a consequence, thrombotic events are frequent in MPN, particularly in PV. Clinically relevant thrombosis is found at time of diagnosis in $11-39 \%$ of PV and in $8-29 \%$ of ET $[56,57]$. Thromboses often also complicate the further course of MPN as seen in $8-19 \%$ of PV and $8-31 \%$ of ET patients and outweigh the risk of bleeding complications, which also typically occur in MPN patients, but at lower frequencies than thromboses [19]. Thus, thromboses are a substantial contributor to symptom burden of MPN patients and impact on their life expectancies [58] as demonstrated by the largest epidemiologic study in PV (European Collaboration on Low dose Aspirin in Polycythemia vera, ECLAP), which showed cardiovascular events to account for $41 \%$ of all deaths [59]. The arterial circulation is more prone to thrombosis in MPN accounting for $60-70 \%$ of events mostly as cerebro- or cardiovascular complications and less frequently as peripheral arterial occlusions. Venous thromboembolism accounts for the remainder with a high incidence of thrombosis in atypical locations such as cerebral venous sinuses and splanchnic veins including mesenteric, portal, and hepatic vein thrombosis (Budd-Chiari syndrome). As MPN represent the most common cause of splanchnic vein thromboses, such events should always raise a high suspicion of underlying MPN [17].

As thrombosis related to HIT has been reported in patients with PV or ET (Table 1), we hypothesized that an immune response to PF4 may be relevant in MPN with a potential implication in thrombotic events. Strong predictors of thrombotic complications in MPN have long been known as a history of previous thrombosis and advanced age $>60$ years [22, 24, 26-30]. General risk factors for arterial or venous thrombosis such as cardiovascular parameters and hereditary thrombophilia, respectively, are prevalent also in MPN patients and appear to mediate additive prothrombotic 
propensity [17]. In addition, multiple MPN-specific factors promoting thrombosis have been identified recently. They comprise both quantitative and qualitative alterations of cellular blood components including erythrocytes, leukocytes, and platelets and extend to functional changes of the endothelium and the hemostatic cascade, thus mediating an overall hypercoagulable state. It has also been established that the presence of the JAK2V617F mutation associates with increased thrombotic potential, whereas the role of the actual $J A K 2 \mathrm{~V} 617 \mathrm{~F}$ allele burden remains controversial. The recently developed international prognostic score for risk of thrombosis in ET (IPSET-thrombosis) is implementing advanced age, previous thrombosis, JAK2V617F, and cardiovascular risk factors [25]. Although not considered by the IPSET scoring, leukocytosis and increased hematocrit represent recognized and relevant promoting factors of thrombosis acting mainly via increased blood viscosity, platelet activation through leukocyte-platelet interactions, and secretion of microparticles of activated leukocytes [60]. Elevated platelet count has not been validated as an independent contributing factor, but functional changes have been observed. MPN platelets show increased biosynthesis of thromboxane A2 [61] and the circulating platelet pool in PV and ET is enriched in immature platelets which circulate in an activated state with increased expression of P-selectin and tissue factor [61, 62]. It has been found that platelets in PV and ET, particularly if carrying the JAK2V617F mutation, show increased potential of thrombin generation [63,64]. Of note, platelet activation products such as $\mathrm{PF} 4$ are increased in plasma of MPN patients [63], which could facilitate an anti-PF4 immune response based on increased abundance of antigen. This process could be even more pronounced in patients not on low dose aspirin, which very efficiently reduces thromboxane A2 biosynthesis already at low doses and counteracts platelet activation [61]. Other factors like platelet-leukocyte aggregates, increased production of inflammatory cytokines and reactive oxygen species (ROS), and increased circulating endothelial cells as well as lower levels of protein C illustrate the broad variety of factors implicated in thrombosis in MPN [17]. Functional studies will be needed to determine the significance of their contribution to overt thrombosis in MPN patients. In addition, the broad range of proaggregatory and procoagulatory factors promoting thrombosis formation in MPN is probably not exclusive and additional, yet unidentified factors may further contribute to the complex pathogenesis of multifactorial thromboses in MPN.

Anti-PF4/heparin antibodies as seen in HIT could represent an additional prothrombotic factor in MPN. Several case reports of HIT occurring upon heparin treatment in PV and ET have been published suggesting that patients with MPN might be at risk for HIT-related immune responses and potentially thrombotic events $[41,42,54]$. HIT is a severe, prothrombotic condition facilitating arterial or venous thromboses triggered by antibody formation against PF4/heparin complexes upon treatment with heparin [31]. Diagnostic criteria of HIT include onset of thrombocytopenia within 5-14 days of heparin exposure along with detection of plateletactivating anti-PF4/heparin antibodies, while overt thrombosis is seen in up to $50 \%$ of cases [65]. However, these diagnostic criteria are hampered in PV and ET by baseline thrombocytosis which may mask thrombocytopenia and by the fact that thrombosis in MPN is obviously multifactorial [41, 42]. Insight into the pathogenesis of HIT has been gained which could provide links to a role of antiPF4/heparin antibodies in PV and ET. Crystallization studies have shown that negatively charged heparin is complexing with tetramers of the cationic platelet factor 4 (PF4) after release from activated platelets. Linearization of heparin molecules allows multiple PF4 tetramers to bind, building substantial amounts of PF4/heparin antigen with neoepitopes on PF4 emerging under specific conditions [66]. Formation of anti-PF4/heparin antibodies is induced probably via recognition of PF4/heparin complexes by toll-like receptors [67], and PF4/heparin antibody complexes mediate activation of platelets via binding to the surface Fc $\gamma$ RIIA receptor. Activation of monocytes via binding of PF4/heparin complexes to $\mathrm{F} c \gamma$ RIIA and $\mathrm{F} c \gamma \mathrm{RI}$ enhances thrombin formation by increased tissue factor expression, which further promotes antibody formation by increasing platelet activation and PF4 levels $[68,69]$. Fc $\gamma$ RIIA and Fc $\gamma$ RIIIA are involved in clearance of activated platelets mediating platelet depletion in HIT. Polymorphism in exon 4 of the Fc $\gamma$ RIIA, H131R ( $c 519 \mathrm{G}>\mathrm{A}$; rs1801274) associated with a histidine to arginine substitution, is known to infer a higher risk for overt thrombosis in patients with HIT homozygous for the 131RR allele due to increased platelet activation and tissue factor expression [69]. Also, Fc $\gamma$ RIIA 131RR homozygous patients show reduced inhibitory effects by normal immunoglobulins of IgG2 subtype on Fc $\gamma$ RIIA due to decreased affinity of Fc $\gamma$ RIIA 131RR for IgG2 [69]. In addition to platelet activation via binding of anti-PF4/heparin antibodies to Fc $\gamma$ RIIA via their Fc part, anti-PF4/heparin antibodies have also been found to bind PF4 complexed with endogenous GAGs on platelet, monocyte, and endothelial cell surfaces via their Fab domains [67].

From studies so far, it is evident that heparin represents the major trigger of the anti-PF4 immune response in HIT and shows differential immunogenicity for distinct types of heparin. Exposure to unfractionated heparin (UFH) provokes HIT in $0.3-3 \%$ [32], while frequencies are lower at $0.2-0.8 \%$ on low molecular weight heparin [70]. A study on minimized heparin use showed reductions in anti-PF4/heparin antibody positivity and clinical HIT in hospitalized patients, which highlights heparin exposure as the central inducing factor [71]. Additional factors supporting the development of anti-PF4/heparin antibodies and HIT have been described. Upon heparin exposure, trauma and surgery were associated with increased risk for HIT as compared to general internal medicine patients. This higher propensity for anti-PF4/heparin immune responses in surgical patients was related to tissue damage which could mediate release of glycosaminoglycans (GAG) as endogenous heparin-like polysaccharides and promote platelet activation with higher levels of PF4 in plasma [31] facilitating anti-PF4/heparin antibody formation due to increased availability of antigen. Inflammatory stimuli related to surgical tissue damage could further enhance the immune response to PF4/heparin complexes $[37,38]$. This concept was supported by increased IL6 
levels in patients after cardiovascular surgery with high-titer anti-PF4/heparin antibodies [72]. Also, the inflammatory milieu in the setting of bacterial infections has been shown to enhance anti-PF4/heparin antibody formation and HIT $[35,36]$. PF4/heparin antibody formation may also be facilitated in other conditions characterized by elevated plasmatic PF4 levels such as diabetes [73], atherosclerosis [74], and cardiovascular [75] and renal disease [76]. A study on diabetic patients with increased vascular risk found anti-PF4/heparin antibodies more frequently than in nondiabetic patients [77]. Similar findings have been reported for patients with acute coronary syndrome (ACS) $[78,79]$ or cardiac surgery. Of note, positivity for anti-PF4/heparin antibodies has been associated with increased complication rate and mortality in cardiovascular patients [80].

Interestingly, one study suggested that inflammatory stimuli and platelet activation due to tissue injury upon joint replacement surgery would suffice to evoke anti-PF4/heparin antibodies in the absence of heparin treatment $[39,40]$. AntiPF4/heparin antibodies were detected in $6.5 \%$ of patients with arthroplasty in the absence of heparin and in $15 \%$ when dynamic compression as thromboprophylaxis was applied. We hypothesized that a similar constellation could be at play in a nonsurgical setting in patients with PV and ET. Platelet production and turnover is increased in PV and ET patients and it is known that MPN platelets are circulating in an activated state and show prolonged activation as compared to normal platelets. As a consequence, plasma levels of PF4 are increased [17]. Higher abundance of PF4 antigen and platelet activation could increase the susceptibility for anti-PF4/ heparin antibody formation in MPN in the absence of heparin treatment. In addition, it is established that MPN create an inflammatory milieu with increased plasma levels of multiple cytokines including IL6 [81]. Detailed in vivo studies have revealed that excessive inflammatory cytokines in MPN originate from both the malignant clone and nonmutant cells [82]. Substantial disease burden in MPN is attributed to inflammatory symptoms including fatigue, pruritus, and bone and muscle pain. Treatment with the JAK1/2 inhibitor ruxolitinib reduces cytokine levels and inflammatory symptoms in most MPN patients highlighting a relevance of the inflammatory milieu for disease burden [83]. The inflammatory condition in MPN could further increase the probability for anti-PF4/heparin antibody formation in the absence of heparin exposure similarly to the findings in nonheparinized surgical patients [40], thereby increasing the risk of overt HIT in PV and ET.

Evidence of anti-PF4/heparin immune responses in MPN is scarce so far. A limited number of case series and reports of single cases describe the occurrence of HIT in PV and ET, while cases in MF have not been reported to date (Table 1) $[41,42,54]$. It is challenging to determine the significance of HIT for thrombosis in PV and ET based on this limited data. Furthermore, diagnostic work-ups vary among different reports. The presence of anti-PF4/heparin antibodies has not been assessed in all patients and antibody isotype testing was performed in a minority. Therefore, we investigated a large cohort of 127 MPN patients for anti-PF4/heparin antibodies using a systematic diagnostic approach. As no cases of HIT have been reported so far in MF, we focused our analysis on 76 patients with PV and 51 with ET. We applied a 2-step algorithm using first an anti-PF4/heparin antibody screening ELISA for analysis of patients' plasma followed by confirmatory testing. As differential effects of immunoglobulin isotypes for platelet activation and thrombin generation in HIT are known, positive patients were subjected to immunoglobulin isotype testing with a specific anti-PF4/heparin antibody ELISA determining positivity for $\operatorname{IgG}$, IgM, and IgA, respectively. This uniform work-up in a large cohort of MPN patients for the first time provides consistent data on the frequency of anti-PF4/heparin antibody formation in PV and ET.

Antibody screening detected anti-PF4/heparin immunoglobulins in $17.3 \%$ of all MPN patients in the absence of heparin exposure (Table 4). Antibody formation was more frequent in PV than in ET with $21 \%$ and $11.8 \%$ of patients, respectively. These findings are notable, as anti-PF4/heparin antibodies in PV and ET patients without direct heparin exposure occur at a clearly higher frequency than in patients on heparin treatment known to develop antibodies in $0.3-3 \%$ [32]. Anti-PF4/heparin antibodies were also substantially more prevalent in PV and ET than in healthy individuals, in whom detection of anti-PF4/heparin antibodies is rare [84]. Subsequent validation testing confirmed anti-PF4/heparin antibodies in $14.5 \%$ of PV and $9.8 \%$ of ET patients which consolidates that anti-PF4/heparin antibody formation in MPN exceeds the risk in heparin-treated patients (Table 4, Figure 1). Also, anti-PF4/heparin antibody production was sustained as documented in a subset of PV and ET patients with serial testing at average intervals of 568 days for anti$\mathrm{PF} 4 /$ heparin IgG and 660 days for IgM isotypes. A similar susceptibility for anti-PF4/heparin immune responses has been reported in nonheparinized surgical patients with dynamic compression therapy which lends strong support to our findings of prevalent anti-PF4/heparin antibodies independent of direct heparin exposure in nonsurgical, but hematological patients. Although there is no direct tissue damage in PV and ET, increased platelet activation and PF4 plasma levels are characteristic [17] and an inflammatory milieu is prominently present, as shown by clinical trials and experimental animal models $[82,83]$.

For further characterization of the anti-PF4/heparin immune response in MPN, we performed immunoglobulin isotype testing in PV and ET to delineate the proportion of anti-PF4/heparin IgG with an established role in platelet activation via Fc $\gamma$ RIIA [31]. Anti-PF4/heparin IgG were detected in $9.2 \%$ of $\mathrm{PV}$ patients which exceeds the frequencies seen in heparin-exposed patients [32] or healthy individuals [84] (Table 4, Figure 1). Interestingly, anti-PF4/heparin antibodies in ET patients were of IgM isotype for which a role in pathogenesis of HIT is controversial. As case reports of overt HIT in ET have been published [41, 42, 46-51], but exclusively IgM are detected in our ET cohort, functional effects of IgM should be evaluated in future studies. Isotype specification of anti-PF4/heparin antibodies in our cohort did not identify any IgA-positive patients and none of the patients tested positive for multiple isotypes, whereas studies on the dynamics of anti-PF4/heparin immune responses 
have reported simultaneous detection of $\operatorname{IgG}$, IgM, and IgA [33]. These differences in antibody isotype pattern may relate to differential immune mechanisms involved in anti$\mathrm{PF} 4 /$ heparin immune responses in patients on heparin treatment versus patients with PV and ET.

Correlation with clinical characteristics revealed a tendency for more thrombotic events in PV patients with antiPF4/heparin IgG immunoglobulins. We observed a $31 \%$ increase of relative risk for thrombotic complications in IgGpositive PV patients as compared to IgG-negative PV suggesting that anti-PF4/heparin IgG antibodies could potentially contribute to the multifactorial thromboses occurring in PV. Higher patient numbers would have been required to reach statistical significance of this finding, but the rarity of MPN is impeding the formation of more extensive patient cohorts (Tables 5 and 6). The fact that anti-PF4/heparin antibodies in PV patients with thrombosis were of IgG isotype may support a functional relevance of anti-PF4/heparin immune responses for increased thrombotic risk. Collaborative multicenter efforts will be required to further extend patient numbers even in rare disorders like MPN, to specifically explore the effect of anti-PF4/heparin IgG for overt thrombosis in PV and ET.

Special forms of HIT including spontaneous or delayed onset HIT are being discussed in rare cases. State-ofthe-art diagnostic criteria for spontaneous HIT have been proposed and include thrombocytopenia and thrombosis without previous heparin exposure along with detectable anti-PF4/heparin antibodies with platelet-activating potential in the absence of heparin [85]. Delayed onset HIT may correspondingly occur days to weeks after heparin exposure. However, diagnostic criteria for these particular HIT phenotypes do not correspond well to the situation in PV and ET. Thrombocytopenia is often masked by excessive platelet production and pathogenesis of thromboses is strongly multifactorial. In MPN, it is the finding of surprisingly frequent, endogenous anti-PF4/heparin antibody formation including particularly IgG isotypes in PV in the current study which deserves attention. It should increase our awareness for platelet activation and inflammatory stimuli as contributors to the anti-PF4/heparin immune response in nonsurgical patients even in the absence of heparin exposure. Future collaborative multicenter studies will hopefully allow for analyses of more extensive patient cohorts also in these rare disorders to unequivocally determine the significance of antiPF4/heparin antibodies for thrombotic risk in MPN. Functional studies will be required to characterize the mechanisms of the anti-PF4/heparin immune response in the absence of heparin exposure. Further insight into the functional basis of this phenomenon could inform measures to reduce antiPF4/heparin antibody formation and potential thromboses. Our data demonstrate that PV patients need to be tested for anti-PF4 IgG at the latest when an actual thrombotic event occurs. As anti-PF4 IgG are endogenously prevalent at a substantial frequency in $\mathrm{PV}$, their presence needs to be excluded before treatment with heparin, which could enhance anti-PF4/heparin immune response and thrombotic risk [55], is initiated to facilitate alternative anticoagulation in anti-PF4 IgG-positive PV patients. Prospective studies of anti-PF4/heparin antibody formation in MPN should follow and will lead to more general recommendations for diagnostic and clinical management of PV and ET patients with anti-PF4/heparin antibodies.

\section{Conflicts of Interest}

There are no conflicts of interest to declare.

\section{Acknowledgments}

This work was supported by the University Hospital Basel and by research support to Sara C. Meyer from the Swiss National Science Foundation, the Swiss Cancer League, and the Swiss Society of Hematology and the Miescher Foundation.

\section{References}

[1] D. A. Arber, A. Orazi, R. Hasserjian et al., "The 2016 revision to the World Health Organization classification of myeloid neoplasms and acute leukemia," Blood, vol. 127, no. 20, pp. 23912405,2016

[2] A. Tefferi and A. Pardanani, "Myeloproliferative neoplasms: a contemporary review," JAMA Oncology, vol. 1, no. 1, pp. 97-105, 2015.

[3] R. Rampal, F. Al-Shahrour, O. Abdel-Wahab et al., "Integrated genomic analysis illustrates the central role of JAK-STAT pathway activation in myeloproliferative neoplasm pathogenesis," Blood, vol. 123, no. 22, pp. e123-e133, 2014.

[4] S. C. Meyer and R. L. Levine, "Molecular pathways: molecular basis for sensitivity and resistance to JAK kinase inhibitors," Clinical Cancer Research, vol. 20, no. 8, pp. 2051-2059, 2014.

[5] E. Parganas, D. Wang, D. Stravopodis et al., "Jak2 is essential for signaling through a variety of cytokine receptors," Cell, vol. 93, no. 3, pp. 385-395, 1998.

[6] R. Kralovics, F. Passamonti, A. S. Buser et al., "A gain-of-function mutation of JAK2 in myeloproliferative disorders," The New England Journal of Medicine, vol. 352, no. 17, pp. 1779-1790, 2005.

[7] R. L. Levine, M. Wadleigh, J. Cools et al., "Activating mutation in the tyrosine kinase JAK2 in polycythemia vera, essential thrombocythemia, and myeloid metaplasia with myelofibrosis," Cancer Cell, vol. 7, no. 4, pp. 387-397, 2005.

[8] C. James, V. Ugo, J.-P. Le Couédic et al., "A unique clonal JAK2 mutation leading to constitutive signalling causes polycythaemia vera," Nature, vol. 434, no. 7037, pp. 1144-1148, 2005.

[9] E. J. Baxter, L. M. Scott, and P. J. Campbell, "Acquired mutation of the tyrosine kinase JAK2 in human myeloproliferative disorders," The Lancet, vol. 365, no. 9464, pp. 1054-1061, 2005.

[10] L. M. Scott, W. Tong, R. L. Levine et al., "JAK2 exon 12 mutations in polycythemia vera and idiopathic erythrocytosis," The New England Journal of Medicine, vol. 356, no. 5, pp. 459-468, 2007.

[11] Y. Pikman, B. H. Lee, T. Mercher et al., "MPLW515L is a novel somatic activating mutation in myelofibrosis with myeloid metaplasia," PLoS Medicine, vol. 3, no. 7, article e270, 2006.

[12] J. Nangalia, C. E. Massie, E. J. Baxter et al., "Somatic CALR mutations in myeloproliferative neoplasms with nonmutated JAK2," The New England Journal of Medicine, vol. 369, no. 25, pp. 2391-2405, 2013. 
[13] T. Klampfl, H. Gisslinger, A. S. Harutyunyan et al., "Somatic mutations of calreticulin in myeloproliferative neoplasms," The New England Journal of Medicine, vol. 369, no. 25, pp. 23792390, 2013.

[14] C. Marty, C. Pecquet, H. Nivarthi et al., "Calreticulin mutants in mice induce an MPL-dependent thrombocytosis with frequent progression to myelofibrosis," Blood, vol. 127, no. 10, pp. 13171324, 2016.

[15] I. Chachoua, C. Pecquet, M. El-Khoury et al., "Thrombopoietin receptor activation by myeloproliferative neoplasm associated calreticulin mutants," Blood, vol. 127, no. 10, pp. 1325-1335, 2016.

[16] R. C. Skoda, A. Duek, and J. Grisouard, "Pathogenesis of myeloproliferative neoplasms," Experimental Hematology, vol. 43, no. 8, pp. 599-608, 2015.

[17] A. Falanga and M. Marchetti, "Thrombosis in myeloproliferative neoplasms," Seminars in Thrombosis and Hemostasis, vol. 40, no. 3, pp. 348-358, 2014.

[18] S. Kreher, S. Ochsenreither, R. U. Trappe et al., "Prophylaxis and management of venous thromboembolism in patients with myeloproliferative neoplasms: consensus statement of the Haemostasis Working Party of the German Society of Hematology and Oncology (DGHO), the Austrian Society of Hematology and Oncology (ÖGHO) and Society of Thrombosis and Haemostasis Research (GTH e.V.)," Annals of Hematology, vol. 93, no. 12, pp. 1953-1963, 2014.

[19] E. Papadakis, R. Hoffman, and B. Brenner, "Thrombohemorrhagic complications of myeloproliferative disorders," Blood Reviews, vol. 24, no. 6, pp. 227-232, 2010.

[20] T. Barbui and A. Falanga, "Molecular biomarkers of thrombosis in myeloproliferative neoplasms," Thrombosis Research, vol. 140, supplement 1, pp. S71-S75, 2016.

[21] A. M. Vannucchi, "From leeches to personalized medicine: evolving concepts in the management of polycythemia vera," Haematologica, vol. 102, no. 1, pp. 18-29, 2017.

[22] R. Marchioli, G. Finazzi, R. Landolfi et al., "Vascular and neoplastic risk in a large cohort of patients with polycythemia vera," Journal of Clinical Oncology, vol. 23, no. 10, pp. 2224-2232, 2005.

[23] F. Passamonti, E. Rumi, L. Arcaini et al., "Prognostic factors for thrombosis, myelofibrosis, and leukemia in essential thrombocythemia: a study of 605 patients," Haematologica, vol. 93, no. 11, pp. 1645-1651, 2008.

[24] T. Barbui, G. Barosi, G. Birgegard et al., "Philadelphia-negative classical myeloproliferative neoplasms: critical concepts and management recommendations from European leukemiaNet," Journal of Clinical Oncology, vol. 29, no. 6, pp. 761-770, 2011.

[25] T. Barbui, G. Finazzi, A. Carobbio et al., "Development and validation of an International Prognostic Score of thrombosis in World Health Organization-essential thrombocythemia (IPSET-thrombosis)," Blood, vol. 120, no. 26, pp. 5128-5252, 2012.

[26] S. Cortelazzo, Viero P., Finazzi G., D’Emilio A., Rodeghiero F., and Barbui T., "Incidence and risk factors for thrombotic complications in a historical cohort of 100 patients with essential thrombocythemia," Journal of Clinical Oncology, vol. 8, no. 3, pp. 556-562, 1990.

[27] A. P. Wolanskyj, S. M. Schwager, R. F. McClure, D. R. Larson, and A. Tefferi, "Essential thrombocythemia beyond the first decade: life expectancy, long-term complication rates, and prognostic factors," Mayo Clinic Proceedings, vol. 81, no. 2, pp. 159-166, 2006.
[28] C. Besses, F. Cervantes, A. Pereira et al., "Major vascular complications in essential thrombocythemia: a study of the predictive factors in a series of 148 patients," Leukemia, vol. 13, no. 2, pp. 150-154, 1999.

[29] C.-S. Chim, Y.-L. Kwong, A. K.-W. Lie et al., "Long-term outcome of 231 patients with essential thrombocythemia: prognostic factors for thrombosis, bleeding, myelofibrosis, and leukemia," Archives of Internal Medicine, vol. 165, no. 22, pp. 26512658, 2005.

[30] M. Bazzan, G. Tamponi, P. Schinco et al., "Thrombosis-free survival and life expectancy in 187 consecutive patients with essential thrombocythemia," Annals of Hematology, vol. 78, no. 12, pp. 539-543, 1999.

[31] O. Onwuemene and G. M. Arepally, "Heparin-induced thrombocytopenia: research and clinical updates," Hematology. American Society of Hematology. Education Program, vol. 2016, no. 1, pp. 262-268, 2016.

[32] T. E. Warkentin, M. N. Levine, J. Hirsh et al., "Heparin-induced thrombocytopenia in patients treated with low-molecularweight heparin or unfractionated heparin," The New England Journal of Medicine, vol. 332, no. 20, pp. 1330-1335, 1995.

[33] A. Greinacher, T. Kohlmann, U. Strobel, J. A. I. Sheppard, and T. E. Warkentin, "The temporal profile of the anti-PF4/heparin immune response," Blood, vol. 113, no. 20, pp. 4970-4976, 2009.

[34] N. Martel, J. Lee, and P. S. Wells, "Risk for heparin-induced thrombocytopenia with unfractionated and low-molecular-weight heparin thromboprophylaxis: a meta-analysis," Blood, vol. 106, no. 8, pp. 2710-2715, 2005.

[35] A. Greinacher, B. Holtfreter, K. Krauel et al., "Association of natural anti-platelet factor 4/heparin antibodies with periodontal disease," Blood, vol. 118, no. 5, pp. 1395-1401, 2011.

[36] K. Krauel, C. Pötschke, C. Weber et al., "Platelet factor 4 binds to bacteria, inducing antibodies cross-reacting with the major antigen in heparin-induced thrombocytopenia," Blood, vol. 117, no. 4, pp. 1370-1378, 2011.

[37] T. E. Warkentin, J.-A. I. Sheppard, C. S. Sigouin, T. Kohlmann, P. Eichler, and A. Greinacher, "Gender imbalance and risk factor interactions in heparin-induced thrombocytopenia," Blood, vol. 108, no. 9, pp. 2937-2941, 2006.

[38] N. Lubenow, P. Hinz, S. Thomaschewski et al., "The severity of trauma determines the immune response to PF4/heparin and the frequency of heparin-induced thrombocytopenia," Blood, vol. 115, no. 9, pp. 1797-1803, 2010.

[39] T. E. Warkentin, "Knee replacement and HIT without heparin," Blood, vol. 127, no. 8, pp. 961-962, 2016.

[40] S. Bito, S. Miyata, K. Migita et al., "Mechanical prophylaxis is a heparin-independent risk for anti-platelet factor 4/heparin antibody formation after orthopedic surgery," Blood, vol. 127, no. 8, pp. 1036-1043, 2016.

[41] M. L. Randi, F. Tezza, M. Scapin et al., "Heparin-induced thrombocytopenia in patients with philadelphia-negative myeloproliferative disorders and unusual splanchnic or cerebral vein thrombosis," Acta Haematologica, vol. 123, no. 3, pp. 140-145, 2010.

[42] G. Spectre, Y. Kalish, L. Schliamser, and D. Varon, "Heparininduced thrombocytopenia in myeloproliferative disorders: a rare or under-diagnosed complication?" American Journal of Hematology, vol. 83, no. 5, pp. 420-423, 2008.

[43] T. Hayashi, Y. Suyama, M. Kaneko et al., "Heparin-induced thrombocytopenia and thrombosis in a patient with polycythemia vera," Internal Medicine, vol. 43, no. 7, pp. 587-589, 2004. 
[44] S. Garcia, A. Cervero, M. Sanchez, and P. Colomina, "Heparinassociated thrombocytopenia in a patient with polycythemia vera: the importance of a marked drop in platelet count," Acta Haematologica, vol. 85, no. 3, pp. 169-170, 1991.

[45] A. P. Kyritsis, E. C. Williams, and H. S. Schutta, "Cerebral venous thrombosis due to heparin-induced thrombocytopenia," Stroke, vol. 21, no. 10, pp. 1503-1505, 1990.

[46] D. S. Houston, "Heparin-induced thrombocytopenia without thrombocytopenia in a patient with essential thrombocythemia," American Journal of Hematology, vol. 65, no. 4, pp. 331$332,2000$.

[47] L. Risch, H. Pihan, C. Zeller, and A. R. Huber, "ET gets HITthrombocytotic heparin-induced thrombocytopenia (HIT) in a patient with essential thrombocythemia (ET)," Blood Coagulation and Fibrinolysis, vol. 11, no. 7, pp. 663-667, 2000.

[48] E. U. Walther, F. P. Tiecks, and R. L. Haberl, "Cranial sinus thrombosis associated with essential thrombocythemia followed by heparin-associated thrombocytopenia," Neurology, vol. 47, no. 1, pp. 300-301, 1996.

[49] M. Lapecorella, A. Lucchesi, M. Di Ianni et al., "Unusual onset of venous thromboembolism and heparin-induced thrombocytopenia in a patient with essential thrombocythemia," Blood Coagulation and Fibrinolysis, vol. 21, no. 1, pp. 85-90, 2010.

[50] S. Richard, J. Perrin, K. Lavandier, J.-C. Lacour, and X. Ducrocq, "Cerebral venous thrombosis due to essential thrombocythemia and worsened by heparin-induced thrombocytopenia and thrombosis," Platelets, vol. 22, no. 2, pp. 157-159, 2011.

[51] A. Murawaki, H. Nakayasu, M. Doi et al., "Heparin-induced thrombocytopenia in essential thrombocytosis," Journal of Stroke and Cerebrovascular Diseases, vol. 21, no. 8, pp. 916.e1916.e5, 2012.

[52] R. Akoum, D. Mahfoud, A. Ghaoui et al., "Budd-Chiari syndrome and heparin-induced thrombocytopenia in polycythemia vera: successful treatment with repeated TIPS and intrferon alpha," Journal of Cancer Research and Therapeutics, vol. 5, no. 4, pp. 305-308, 2009.

[53] E. Biagioni, P. Pedrazzi, M. Marietta et al., "Successful liver transplantation in a patient with splanchnic vein thrombosis and pulmonary embolism due to polycythemia vera with Jak $2 \mathrm{v} 617 \mathrm{f}$ mutation and heparin-induced thrombocytopenia," Journal of Thrombosis and Thrombolysis, vol. 36, no. 3, pp. 352-354, 2013.

[54] J. Bovet, E. De Maistre, Y. Bejot, and F. Girodon, "Are myeloproliferative neoplasms a risk factor for heparin-induced thrombocytopenia?" British Journal of Haematology, vol. 175, pp. 537-539, 2016.

[55] T. E. Warkentin, M. Makris, R. M. Jay, and J. G. Kelton, "A spontaneous prothrombotic disorder resembling heparin-induced thrombocytopenia," The American Journal of Medicine, vol. 121, no. 7, pp. 632-636, 2008.

[56] A. Tefferi and M. Elliott, "Thrombosis in myeloproliferative disorders: prevalence, prognostic factors, and the role of leukocytes and JAK2V617F," Seminars in Thrombosis and Hemostasis, vol. 33, no. 4, pp. 313-320, 2007.

[57] A. Casini, P. Fontana, and T. P. Lecompte, "Thrombotic complications of myeloproliferative neoplasms: risk assessment and risk-guided management," Journal of Thrombosis and Haemostasis, vol. 11, no. 7, pp. 1215-1227, 2013.

[58] A. Falanga and M. Marchetti, "Thrombotic disease in the myeloproliferative neoplasms," Hematology. American Society of Hematology. Education Program, vol. 2012, pp. 571-581, 2012.
[59] R. Landolfi, R. Marchioli, J. Kutti et al., "Efficacy and safety of low-dose aspirin in polycythemia vera," The New England Journal of Medicine, vol. 350, no. 2, pp. 114-124, 2004.

[60] C. M. Hobbs, H. Manning, C. Bennett et al., "JAK2V617F leads to intrinsic changes in platelet formation and reactivity in a knock-in mouse model of essential thrombocythemia," Blood, vol. 122, no. 23, pp. 3787-3797, 2013.

[61] R. Landolfi, G. Ciabattoni, P. Patrignani et al., "Increased thromboxane biosynthesis in patients with polycythemia vera: evidence for aspirin-suppressible platelet activation in vivo," Blood, vol. 80, no. 8, pp. 1965-1971, 1992.

[62] E. Arellano-Rodrigo, A. Alvarez-Larrán, J. C. Reverter, N. Villamor, D. Colomer, and F. Cervantes, "Increased platelet and leukocyte activation as contributing mechanisms for thrombosis in essential thrombocythemia and correlation with the JAK2 mutational status," Haematologica, vol. 91, no. 2, pp. 169-175, 2006.

[63] M. K. Jensen, P. de Nully Brown, B. V. Lund, O. J. Nielsen, and H. C. Hasselbalch, "Increased platelet activation and abnormal membrane glycoprotein content and redistribution in myeloproliferative disorders," British Journal of Haematology, vol. 110, no. 1, pp. 116-124, 2000.

[64] M. Panova-Noeva, M. Marchetti, H. M. Spronk et al., "Plateletinduced thrombin generation by the calibrated automated thrombogram assay is increased in patients with essential thrombocythemia and polycythemia vera," American Journal of Hematology, vol. 86, no. 4, pp. 337-342, 2011.

[65] T. E. Warkentin, "Heparin-induced thrombocytopenia: pathogenesis and management," British Journal of Haematology, vol. 121, no. 4, pp. 535-555, 2003.

[66] Z. Cai, S. V. Yarovoi, Z. Zhu et al., "Atomic description of the immune complex involved in heparin-induced thrombocytopenia," Nature Communications, vol. 6, p. 8277, 2015.

[67] M. M. Prechel and J. M. Walenga, "Emphasis on the Role of PF4 in the Incidence, Pathophysiology and Treatment of Heparin Induced Thrombocytopenia," Thrombosis Journal, vol. 11, no. 1, article no. 7, 2013.

[68] V. Tutwiler, D. Madeeva, H. S. Ahn et al., "Platelet transactivation by monocytes promotes thrombosis in heparin-induced thrombocytopenia," Blood, vol. 127, no. 4, pp. 464-472, 2016.

[69] J. Rollin, C. Pouplard, and Y. Gruel, "Risk factors for heparininduced thrombocytopenia: focus on Fcgamma receptors," Thromb Haemost, vol. 16, pp. 799-805, 2016.

[70] P. Prandoni, S. Siragusa, B. Girolami, and F. Fabris, "The incidence of heparin-induced thrombocytopenia in medical patients treated with low-molecular-weight heparin: a prospective cohort study," Blood, vol. 106, no. 9, pp. 3049-3054, 2005.

[71] K. E. McGowan, J. Makari, A. Diamantouros et al., "Clinical trials and observations: reducing the hospital burden of heparin-induced thrombocytopenia: Impact of an avoid-heparin program," Blood, vol. 127, no. 16, pp. 1954-1959, 2016.

[72] D. Paparella, G. Scrascia, A. Galeone et al., "Formation of antiplatelet factor 4/heparin antibodies after cardiac surgery: influence of perioperative platelet activation, the inflammatory response, and histocompatibility leukocyte antigen status," Journal of Thoracic and Cardiovascular Surgery, vol. 136, no. 6, pp. 14561463, 2008.

[73] G. Davì, P. Gresele, F. Violi et al., "Diabetes mellitus, hypercholesterolemia, and hypertension but not vascular disease per se are associated with persistent platelet activation in vivo: evidence derived from the study of peripheral arterial disease," Circulation, vol. 96, no. 1, pp. 69-75, 1997. 
[74] S. Pitsilos, J. Hunt, E. R. Mohler et al., "Platelet factor 4 localization in carotid atherosclerotic plaques: correlation with clinical parameters," Thrombosis and Haemostasis, vol. 90, pp. 1112-1120, 2003.

[75] F. Laghrissi-Thode, W. R. Wagner, B. G. Pollock, P. C. Johnson, and M. S. Finkel, "Elevated platelet factor 4 and $\beta$-thromboglobulin plasma levels in depressed patients with ischemic heart disease," Biological Psychiatry, vol. 42, no. 4, pp. 290-295, 1997.

[76] Y. Endo, S. Mamiya, M. Satoh, K. Takahashi, and T. Harada, "Plasma $\beta$-thromboglobulin and platelet factor 4 in patients with chronic renal failure and effect of hemodialysis," The Tohoku Journal of Experimental Medicine, vol. 135, no. 4, pp. 349358, 1981.

[77] J. J. Naoum, N. R. Chamoun, M. S. Patel et al., "Elevated Heparin-Induced Antibodies Are More Common in Diabetic Patients with Vascular Disease," Thrombosis, vol. 2014, Article ID 649652, 5 pages, 2014.

[78] A. V. Mattioli, L. Bonetti, S. Sternieri, and G. Mattioli, "Heparininduced thrombocytopenia in patients treated with unfractionated heparin: prevalence of thrombosis in a 1 year follow-up," Italian Heart Journal, vol. 1, no. 1, pp. 39-42, 2000.

[79] E. Bennett-Guerrero, T. F. Slaughter, W. D. White et al., "Preoperative anti-PF4/heparin antibody level predicts adverse outcome after cardiac surgery," Journal of Thoracic and Cardiovascular Surgery, vol. 130, no. 6, pp. 1567-1572, 2005.

[80] W. K. Stribling, T. F. Slaughter, T. T. Houle, and D. C. Sane, "Beyond the platelet count: heparin antibodies as independent risk predictors," American Heart Journal, vol. 153, no. 6, pp. 900-906, 2007.

[81] A. Tefferi, R. Vaidya, D. Caramazza, C. Finke, T. Lasho, and A. Pardanani, "Circulating interleukin (IL)-8, IL-2R, IL-12, and IL15 levels are independently prognostic in primary myelofibrosis: a comprehensive cytokine profiling study," Journal of Clinical Oncology, vol. 29, no. 10, pp. 1356-1363, 2011.

[82] M. Kleppe, M. Kwak, P. Koppikar et al., "JAK-STAT pathway activation in malignant and nonmalignant cells contributes to MPN pathogenesis and therapeutic response," Cancer Discovery, vol. 5, no. 3, pp. 316-331, 2015.

[83] S. Verstovsek, H. Kantarjian, R. A. Mesa et al., "Safety and efficacy of INCB018424, a JAK1 and JAK2 inhibitor, in myelofibrosis," The New England Journal of Medicine, vol. 363, no. 12, pp. 1117-1127, 2010.

[84] M. J. Hursting, P. J. Pai, J. E. McCracken et al., "Platelet factor 4/heparin antibodies in blood bank donors," American Journal of Clinical Pathology, vol. 134, no. 5, pp. 774-780, 2010.

[85] T. E. Warkentin, P. A. Basciano, J. Knopman, and R. A. Bernstein, "Spontaneous heparin-induced thrombocytopenia syndrome: 2 new cases and a proposal for defining this disorder," Blood, vol. 123, no. 23, pp. 3651-3654, 2014. 


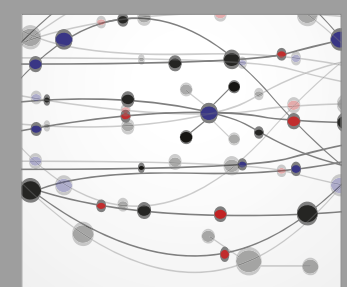

The Scientific World Journal
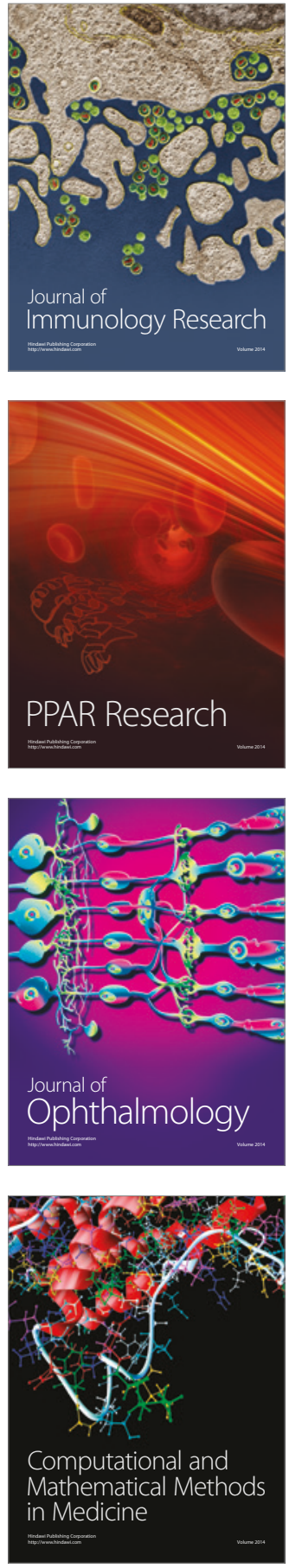

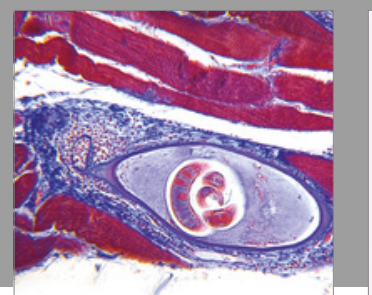

Gastroenterology Research and Practice
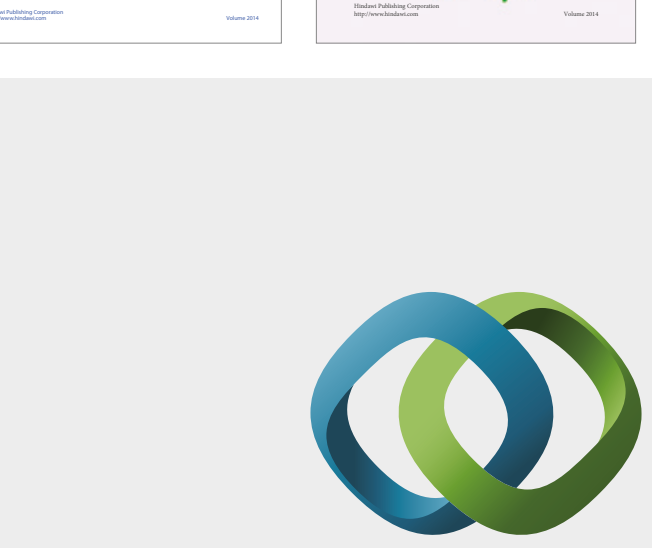

\section{Hindawi}

Submit your manuscripts at

https://www.hindawi.com
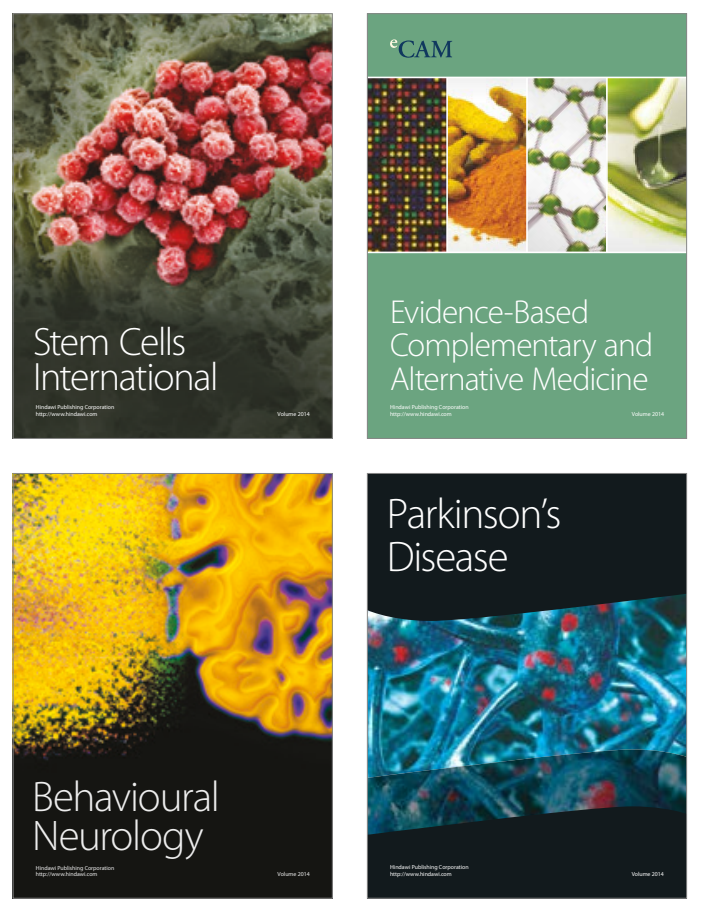
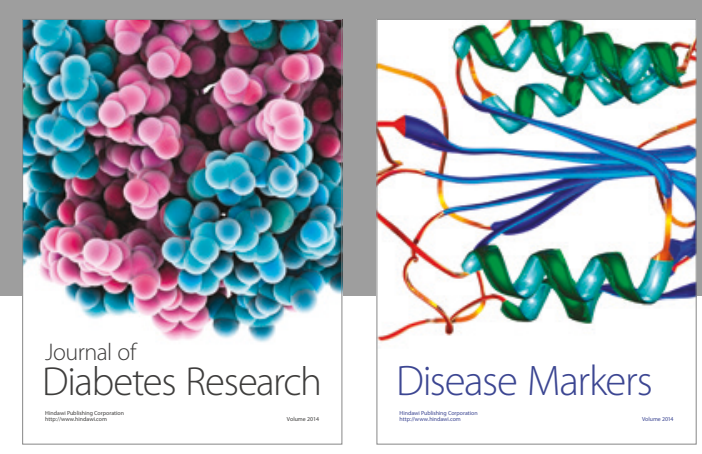

Disease Markers
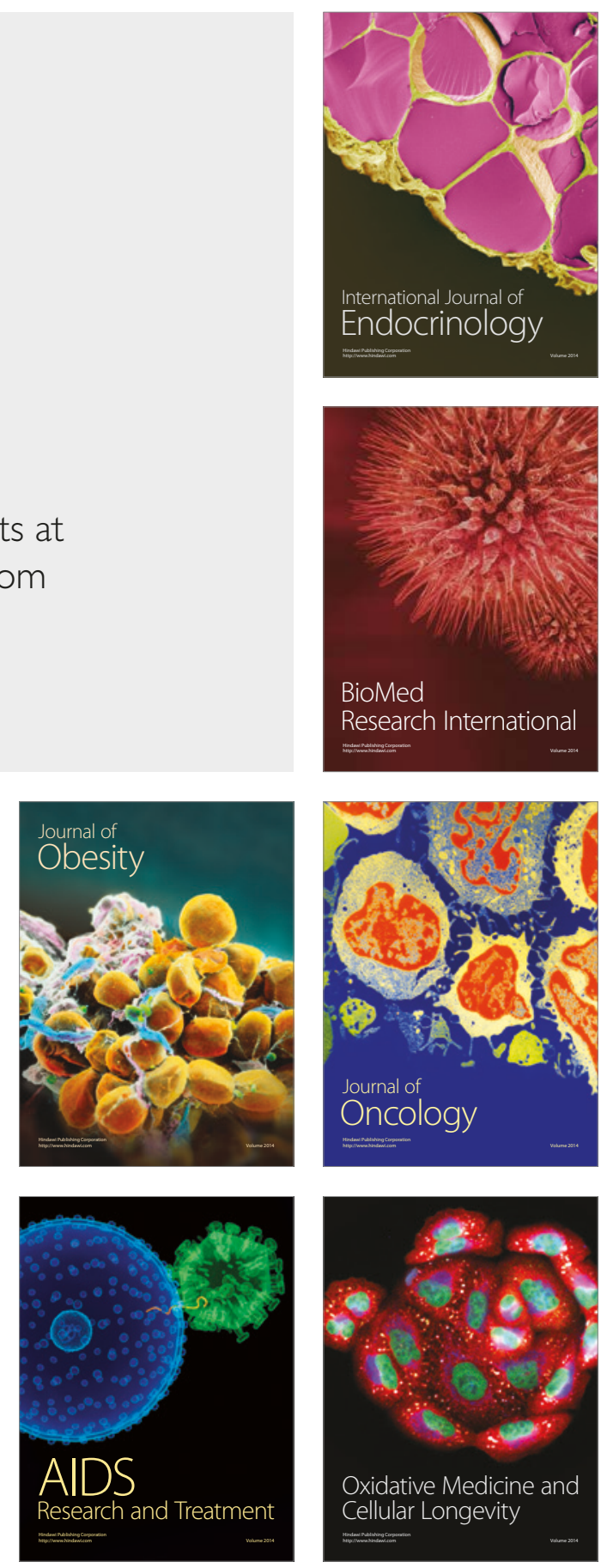